\title{
La grotte Mandrin et les premières occupations du Paléolithique supérieur en Occitanie orientale
}

\author{
LUDOVIC SLIMAK ${ }^{1 *}$ \\ Damien Pesesse ${ }^{1}$ \\ YVES GIRAuD ${ }^{2}$
}

\begin{abstract}
RÉSUMÉ
Le niveau supérieur de la grotte Mandrin présente une courte occupation attribuable au passage d'un groupe humain postérieurement à $35000 \mathrm{BP}$ (Giraud et al. à paraitre). Ces éléments présentent de nettes affinités avec les séries attribuées régionalement à un Aurignacien initial méditerranéen (Bazile 1974, 1999), actuellement reconnu comme la première expression du Paléolithique supérieur dans le sud-est de

la France, région où le Castelperronien n'est pas représenté (Combier 1960, 1967, 1990).

La reconnaissance et la caractérisation d'un faciès de transition non reconnu jusqu'alors est en cours d'élaboration

(Slimak 2004). Située sous un promontoire rocheux dominant la vallée du Rhône au sud de Montélimar (France, Fig. 1), la grotte Mandrin se présente comme un petit abri peu profond ouvert au nord.

Sondée dès 1990, elle livre depuis une importante stratigraphie comprenant huit
\end{abstract}

\section{RESUMEN}

El nivel superior de la Cueva Mandrin indica que fue ocupada por grupos humanos durante un breve periodo después de 35000 BP (Giraud et al., en prensa). La industría leptolitica contenida en este nivel muestra claras afinidades con otras series de la región, atribuidas a un Auriñaciense inicial (Bazile 1974, 1999), actualmente reconocido como la primera expresión del Paleolítico Superior en el sudeste de Francia donde no hay ocupaciones Castelperroniense (Combier 1960, 1967, 1990).

El reconocimiento y la caracterización de una facies de transición, no reconocida hasta la fecha, está en proceso de elaboración (Slimak 2004). Situada en un promontorio rocoso que domina el Valle del Ródano, al sur de Montelimar (fig. 1), la Cueva Mandrin se presenta como un pequeño abrigo, poco profundo y abierto al Norte. Explorada desde 1990, muestra una importante estratigrafia compuesta de 8 niveles atribuibles a un Paleolítico Medio

1 UMR 6636, Economies Sociétés et Environnements Préhistoriques, Maison Méditerranéenne des Sciences de l'Homme, 5 rue du Château de l'Horloge, B.P. 647, 13094 Aix-en-Provence Cedex 2.

* auteur correspondant, corresponding author L. Slimak: slimak@mmsh.univ-aix.fr

2 35, rue espérandieu 13001 Marseille. 
niveaux attribuables à un Paléolithique moyen récent. Au sein du niveau

Protoaurignacien, les lamelles Dufour rectilignes et de grand module occupent

une place prépondérante du système technique. Cette étude permet d'apporter

un nouveau regard sur les systèmes techniques protoaurignaciens et élabore

une synthèse régionale portant sur les premières occupations du Paléolithique supérieur reconnues dans cette région. L'aire géographique concernée par cette synthèse couvre la Provence, le Languedoc oriental, l'Ardèche et la Drôme, qui appartiennent à la frange orientale de l'Occitanie.

\section{MOTS-CLEFS}

Paléolithique supérieur initial, Protoaurignacien, sud-est de la France, matières premières siliceuses, chaines opératoires, grandes lamelles Dufour.
Reciente. En el nivel Protoauriñaciense, las laminitas Dufour de gran tamaño (de más de $5 \mathrm{~cm}$ le longitud) ocupan un papel importante en el sistema técnico.

El presente estudio lanza una nueva mirada sobre los sistemas técnicos y procura esquemas específicos de producción de laminitas. Al mismo tiempo propone una sintesis, fundada en la composición de los conjuntos líticos, de las primeras implantaciones durante el Paleolítico Superior en esta región, que incluye la Provenza, el Languedoc oriental, Ardeche y Drome, todos pertenecientes a la franja oriental de la Occitania.

\section{PALABRAS CLAVES}

Paleolítico superior inicial, Protoauriñaciense, primeras ocupaciones en Occitania, materias primas, cadenas operativas, grandes laminitas Dufour ${ }^{3}$.

\section{CONTEXTE ARCHÉOLOGIQUE}

Le niveau supérieur de la grotte Mandrin s'inscrit dans un horizon caillouteux, mêlé à une matrice fine limoneuse de couleur brun à brun sombre. Cette matrice augmente vers la base (niv 2) et un passage perturbé amène une couche sablolimoneuse jaunâtre recelant les niveaux 3 et 4 (Fig. 2). Les travaux sur la répartition spatiale du matériel archéologique des 4 niveaux supérieurs ont permi d'une part de définir leurs limites verticales et de confirmer les observations faites à la fouille concernant la localisation des débitages lamellaires dans la partie supérieure du niveau 1 (Yvorra et Slimak, 2001, Yvorra, 2003). Les éléments protoaurignaciens sont associés dans la partie supérieure du niveau 1 à un matériel moustérien qui témoigne à la fois de quelques remaniements, notamment en partie occidentale de l'abri $(E-F / 2$ et $E-F / 1,0,-1)$ et de phénomènes de palimpsestes. Ce niveau repose directement sous un horizon holocène et se trouve localement perturbé par une fosse sépulcrale de l'ge du Bronze ancien (LY6106=3880 $\pm 105 \mathrm{BP})$. Une fraction du matériel aurignacien provient du remaniement généré par ces sépultures. L'aplomb actuel du porche a subi un important retrait avant de prendre sa configuration actuelle ce qui a eu pour effet une érosion du remplissage au Nord, entraînant peut-être l'ablation des horizons archéologiques.

Des datations en ATL effectuées par $\mathrm{H}$. Valladas sur silex brûlé provenant de la base du niveau 1 et du sommet du niveau 2 situent chronologiquement les

3 Traductions-translations, Dr Alfredo J. Zamora. Laboratoire ICNE, UMR6544-CNRS. 

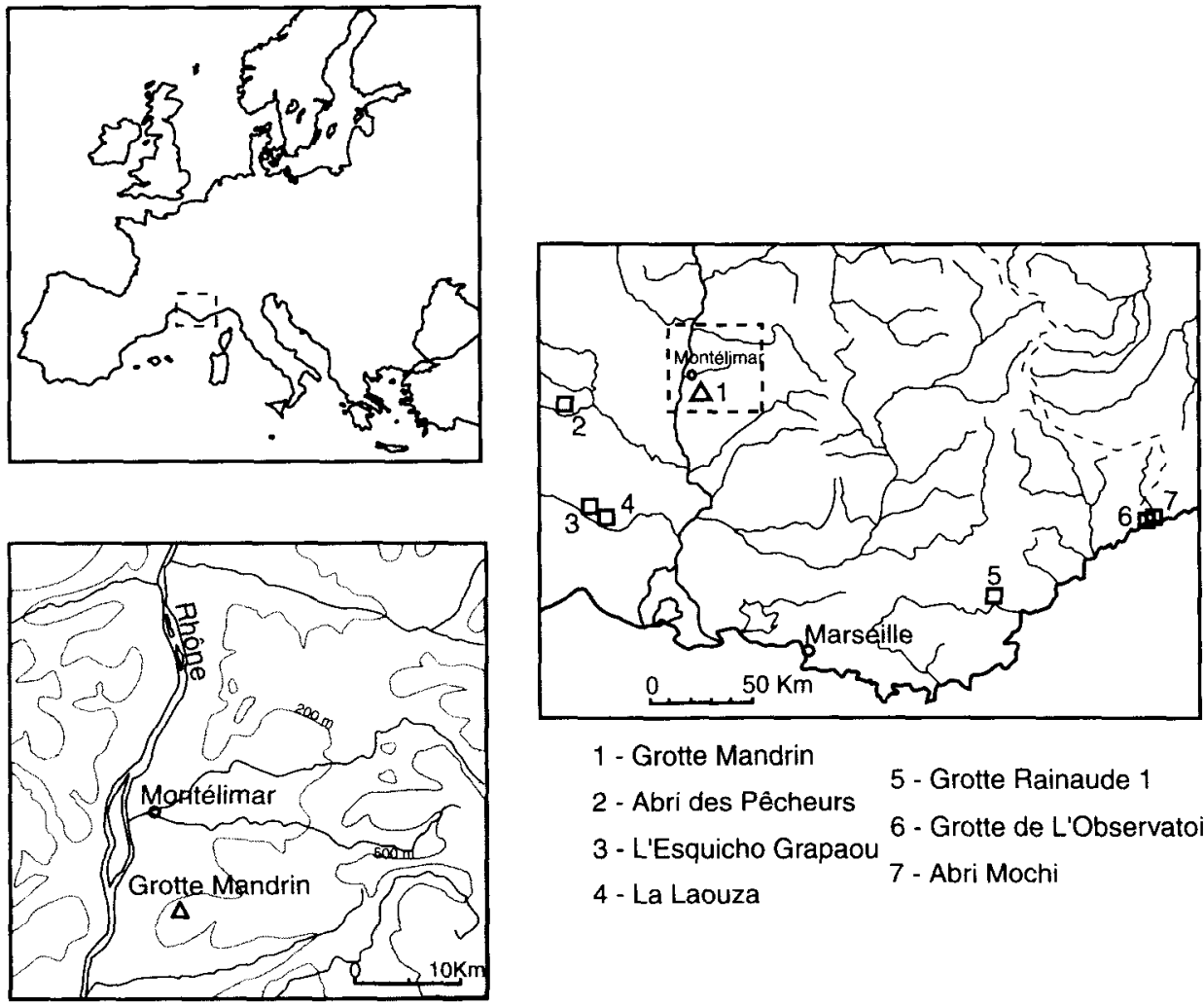

1 - Grotte Mandrin

2 - Abri des Pêcheurs

5 - Grotte Rainaude 1

3 - L'Esquicho Grapaou

6. Grotte de L'Observatoire

4 - La Laouza

7 - Abri Mochi

Figure 1. Situation géographique de la grotte Mandrin et répartition des sites mentionnés dans le texte.

dernières occupations moustériennes autour de 35000 BP en moyenne pondérée (Giraud et al., à paraître). L'ensemble leptolithique étudié ici provient de la partie moyenne et sommitale du niveau 1 , il est donc chronologiquement postérieur aux dates obtenues. Cette position chronologique, postérieure à $35000 \mathrm{BP}$ s'accorde bien avec les datations obtenues dans les gisements protoaurignaciens régionaux ${ }^{4}$. La faune associée correspond plutöt à une association de milieu frais et ouvert, bien que les vestiges fauniques soient peu représentés dans le niveau 1. Pour ce qui concerne les ruminants, le cheval et le bouquetin, suivis du cerf, dominent l'ensemble faunique (Brugal, in: Giraud, 1999). Des éléments de faune plus froide sont représentés par deux restes de Marmotta sp. et un de Rupicapra rupicapra, mais ces taxons ne peuvent attester à eux seuls d'un climat plus rigoureux. L'assemblage archéologique résulte probablement d'un passage de très courte durée, et

4 Esquicho Grapaou niveau CSL1 zone T22: $31850 \pm 1700$ BP (M.C. 1181), et $31850 \pm 1300$ BP (M.C. 1273) (Bazile 1976 a) et niveau SLC1b: $34500 \pm 2000$ BP (Bazile 1999).

Mochi G: $32280 \pm 580 \mathrm{BP}(\mathrm{Oxa}-3588$, Cut 50$)$ et $35700 \pm 850 \mathrm{BP}$ (Oxa -3591 , Cut 59) (Kuhn et al. 1998). 


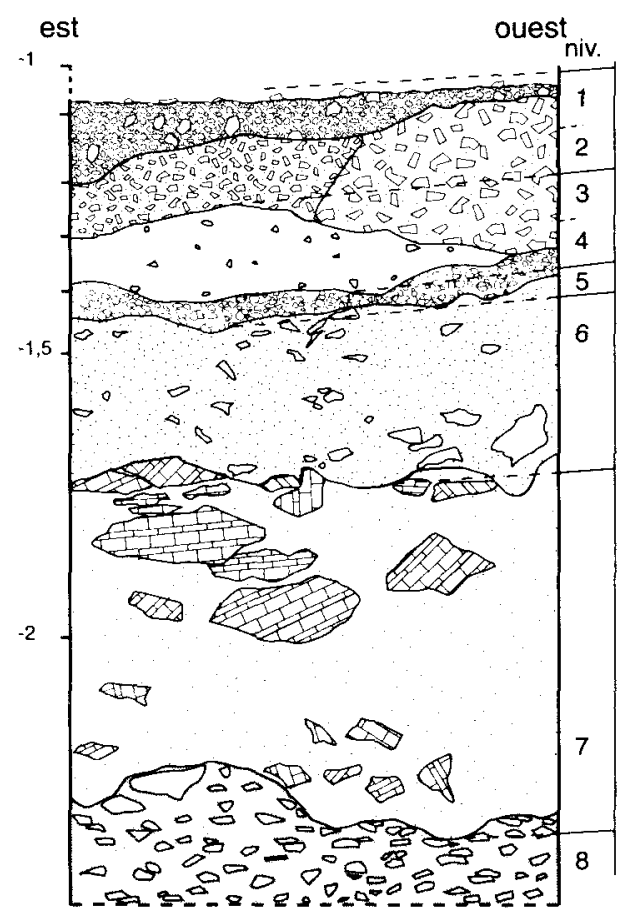

Figure 2. Coupe transversale, sondage 1991, carré G1.

vraisemblablement peu de temps après la dernière occupation du Paléolithique moyen. Les quatre niveaux supérieurs documentent les manifestations moustériennes les plus récentes du sud-est de la France (Slimak, 2004).

\section{LES MATIE்RES PREMIĖRES}

Le matériau utilisé préférentiellement dans la série Protoaurignacienne provient d'un gîte primaire d'accidents siliceux barrémo-bédouliens, situé sur la commune de Malataverne, au lieu-dit Maloubret à environ $2 \mathrm{~km}$ à l'ouest du site (Fig. 4). Ce silex se présente en rognons de formes variées et de taille pluridécimétrique. Sa relation intime avec l'encaissant (calcaires subrécifaux à rudistes de faciès urgonien) entraîne une exploitation privilégiée dans le vallon à l'est du serre de Maloubret où l'on peut encore en trouver. De couleur brun miel à brun plus sombre (S67-69 à T71, parfois S51 (Cailleux)), sa translucidité l'éclaircit et montre une légère pigmentation rouge caractéristique. Les spicules de spongiaires y sont abondants et particulièrement visibles sur les pièces patinées. Le cortex est généralement épais, et envahit la matière siliceuse pure. Des zones plus calcaires, opaques et plus claires parsèment ce matériau qui présente malgré tout une bonne aptitude à la taille. 


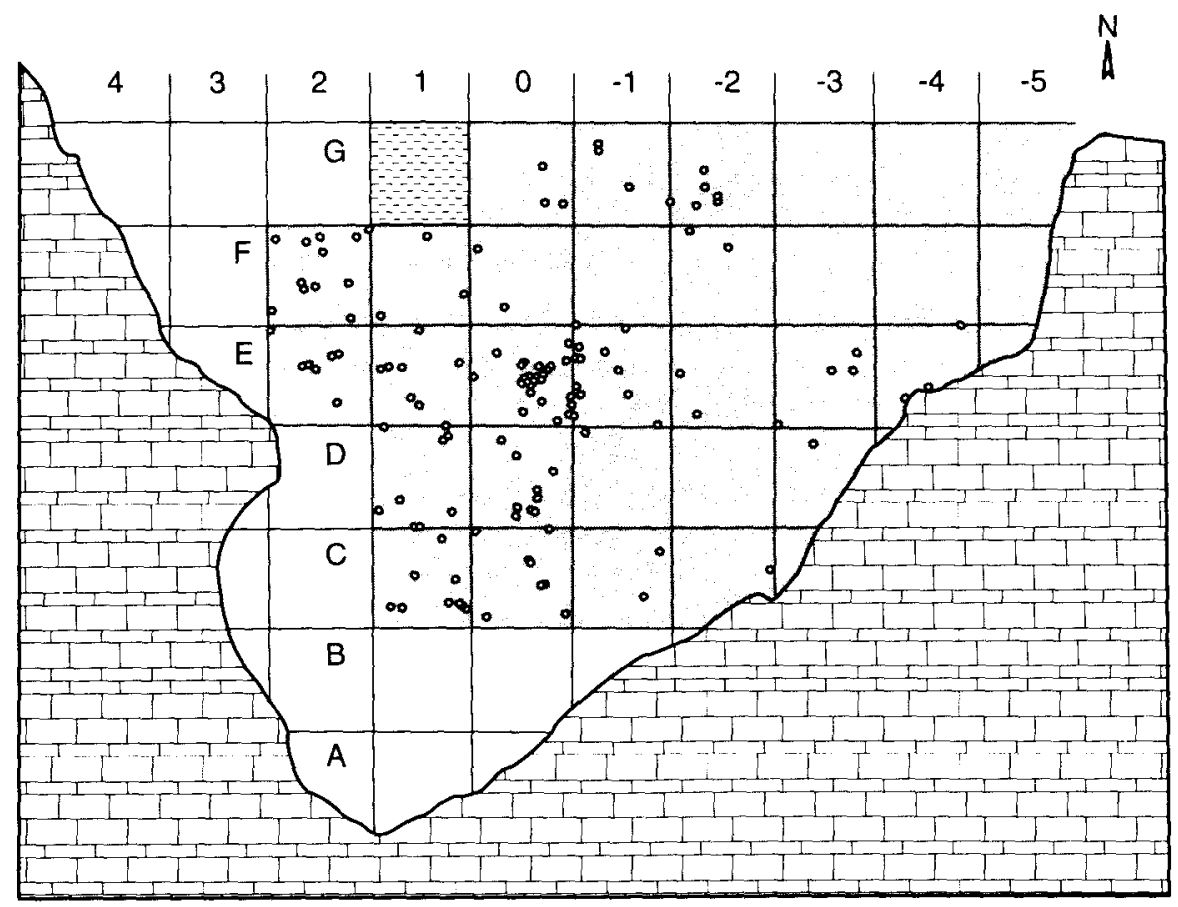

Zone fouillée

Sondage 1991

Eléments aurignaciens

Figure 3. Grotte Mandrin. Répartition des éléments leptolithiques du niveau 1.

L'utilisation de matériaux différents de ce silex de Maloubret est très sporadique. Signalons 4 pièces en silex oligocène, provenant des collines occidentales de Navon et d'affleurements situés à l'est du gisement. La distance aux gîtes n'excède pas $5 \mathrm{~km}$. Une lamelle outrepassée est en silex crétacé brun ardéchois (Les Videaux à Rochemaure) et un fragment de lamelle ainsi qu'un élément entier sont en silex campanien noir d'origine dioise de la haute vallée de la Drôme et du Bez, bien qu'en l'absence de cortex caractéristique, l'origine primaire de cette matière ne soit pas attestée. Une lame à crête est réalisée dans un accident barrémo-bédoulien des falaises dominant le village de Châteauneuf-duRhône, à environ $5 \mathrm{~km}$ de l'abri. Enfin, 4 matières, correspondant à 4 éléments de l'ensemble, n'ont pu être identifiées: un silex gris, un silex beige à tâches, un silex noir translucide (présent sous la forme d'un nucléus, certainement oligocène mais de provenance inconnue) et un accident siliceux en milieu gréseux, peut-être turonien. 


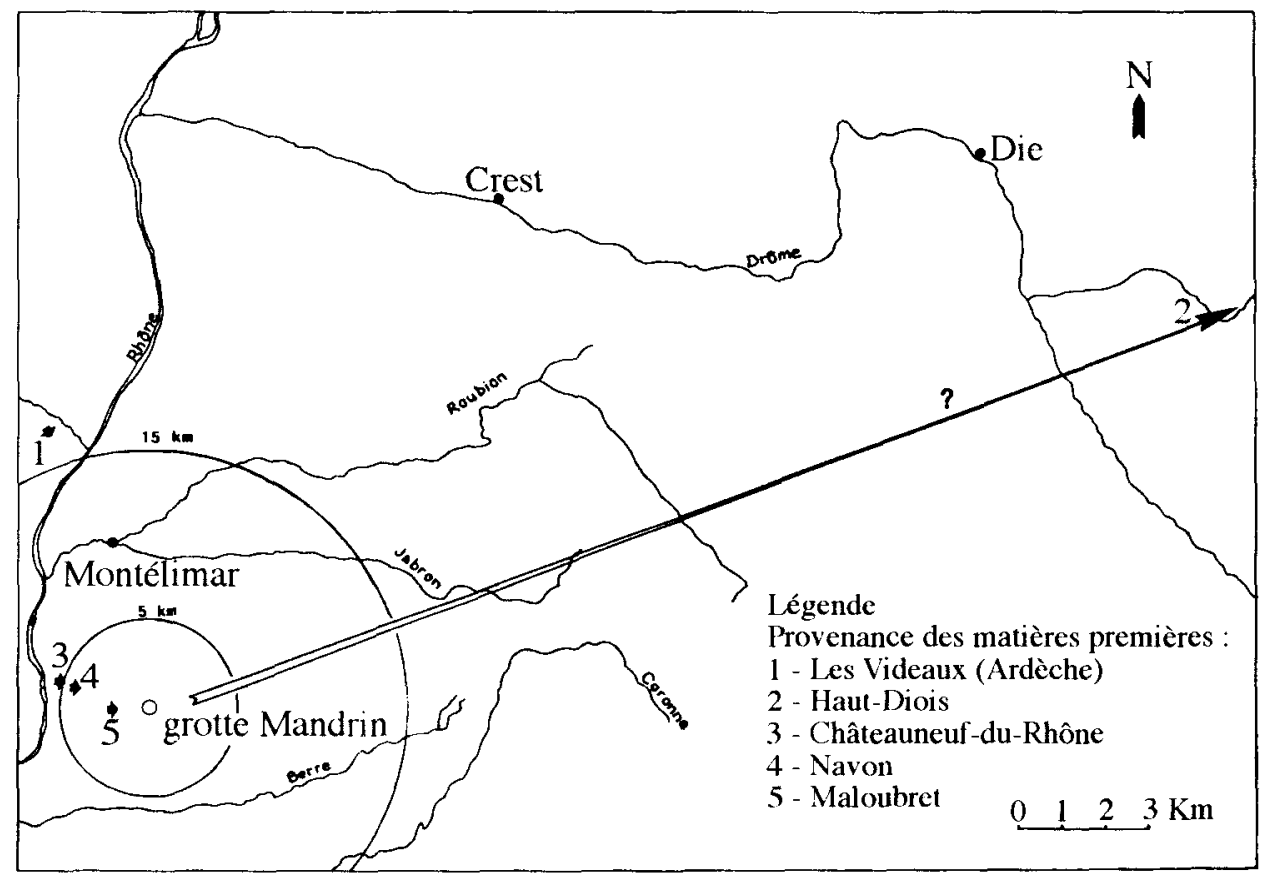

Figure 4. Origine des matières premières des élèments protoaurignaciens de la grotte Mandrin.

II existe par ailleurs quelques pièces chauffées ou trop patinées pour permettre une identification précise du matériau; il s'agit pour certaines de silex barrémobédoulien provenant soit de Maloubret soit des formations ardéchoises.

On constate donc une exploitation particulièrement privilégiée du silex barrémobédoulien de Maloubret et une utilisation plus diffuse de matériaux d'origine somme toute peu lointaine. Seulement un matériau (silex campanien du Diois) pourrait être le fait de déplacements plus importants $(70-80 \mathrm{~km})$. Nous manquons donc malheureusement d'indices pétrographiques déterminants pour appréhender précisément leurs territoires hors environnement immédiat.

En effet, ce constat témoigne d'un comportement particulier d'un groupe de chasseurs en contexte de déplacement, ayant effectué une halte dans la grotte Mandrin après acquisition de matière première et débitage dans l'abri puis départ avec abandon des témoins de leurs activités.

\section{3. ÉTUDE DU MOBILIER}

Cette petite série lithique, composée de 162 artefacts, dégage une nette impression d'homogénéité induite par un faible nombre de blocs exploités. La 
modestie de la collection ne permet pas l'élaboration d'une analyse quantitative solide, en revanche, la forte cohérence de l'assemblage induite par la brièveté de l'occupation permet d'aborder, avec une certaine acuité, les principes techniques régissant l'exploitation des silex. Face à l'effectif de la série, et en dépit de la valeur particulièrement diagnostique d'un certain nombre de pièces, les propositions que nous avons été amené à formuler constituent des bases de travail et non des conclusions définitives.

Les débitages s'articulent essentiellement autour de l'obtention de supports lamellaires, bien que la recherche de produits de plus grand module, laminaires, soit clairement attestée. D'un point de vue technologique, un des points importants est l'individualisation très forte des différents schémas productifs entre eux: débitages laminaires et lamellaires ressortent de dynamiques technologiques fortement dissociées et, nous allons le voir, aucune continuité opératoire ne peut être envisagée entre les schémas laminaires et lamellaires. En revanche, quelques lamelles sont épisodiquement produites au cours de séquences laminaires.

Concernant les débitages lamellaires, il faut immédiatement relever dans cette série la coexistence d'objectifs de production nettement individualisés sous-tendus par des schémas opératoires bien différenciés.

\subsection{Les productions lamellaires}

Trois familles de lamelles peuvent ainsi être individualisées: de petites lamelles rectilignes, dont la longueur ne doit pas excéder $2 \mathrm{~cm}$ entières, pour une largeur gravitant aux alentours de 2 à $3 \mathrm{~mm}$, des lamelles pointues de plus grand module, avoisinant les 3 à $4 \mathrm{~cm}$ de longueur, pour 5 à $6 \mathrm{~mm}$ de largeur, et légèrement convexes et de très grandes lamelles rectilignes dont le gabarit moyen s'articule autour de $6 \mathrm{~cm}$ de longueur pour $1 \mathrm{~cm}$ de largeur.

La première catégorie de petites lamelles semble dévolue à être transformée en produits à dos par retouche directe. Quelques lamelles sont transformées à l'aide de très fines retouches alternes semi-abruptes et très peu transformantes. Les lamelles pointues sont aménagées en lamelles Dufour, sous-type Dufour, et peuvent donc être considérées comme des outils à extrémité distale acuminée. Enfin, les très grandes lamelles pourraient être dévolues à une transformation en outil de la famille Dufour présentant une de leur extrémité resserrée, sans être nécessairement pointue.

La première interrogation porte sur la réalité de ces catégories de lamelles. Leur distinction génère immédiatement une réflexion sur l'éventualité d'une continuité opératoire à l'origine de ces différentes catégories de supports. La réponse ne peut ici être abordée d'un point de vue quantitatif dans la mesure où la série est numériquement modeste. La question peut en revanche être appréhendée en analysant les particularités technologiques de ce système technique. 


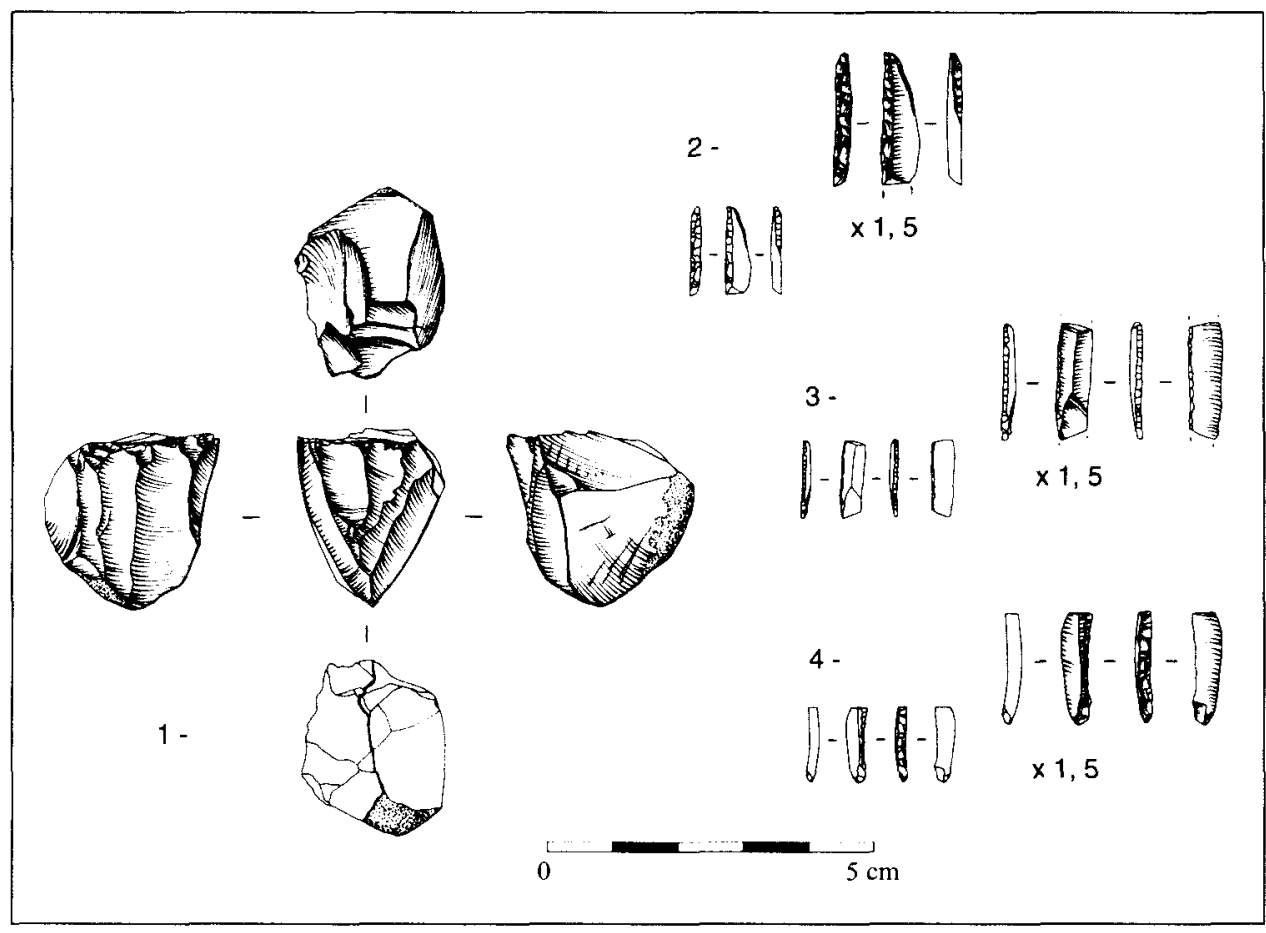

Figure 5. Nucléus à lamelles de petit module (1). pièces à dos $(2,4)$, et lamelle Dufour (3).

Trente-quatre éléments peuvent être rattachés au schéma de petites lamelles (Fig. 5). Ce schéma semble à la fois simple et relativement peu standardisé. Ces petites lamelles rectilignes sont obtenues à partir de l'exploitation de petits éclats dont la tranche ou la face supérieure va être affectée par l'extraction de lamelles suivant un mode globalement semi-tournant. La réalisation de crêtes et de néocrêtes à un versant est attestée par quelques éléments, mais ces crêtes modifient probablement peu la structure générale de l'éclat exploité. En revanche, l'alternance de lamelles de flancs et de lamelles centrées sur la table de débitage a probablement pour finalité une structuration globale du volume de la table de débitage, lui conférant parfois une convergence distale accusée. Cette morphologie serait probablement à corréler avec une recherche de lamelles planes qui présentent globalement une décroissance régulière de leur largeur. Toutefois, ce schéma à petites lamelles rectilignes semble trop peu normé pour pouvoir dégager de fortes récurrences concernant les objectifs des artisans.

II n'en est pas de même en ce qui concerne les lamelles pointues et les grandes lamelles rectilignes qui répondent de comportements productifs fortement normés. Ces débitages s'appuient sur une géométrie des nucléus particulièrement investie par l'artisan. 
Trente pièces peuvent être rattachées au schéma de lamelles pointues. Ces débitages sont réalisés à partir d'éclats, présentant un volume initial nettement plus important que pour la production de petites lamelles. Les objectifs du débitage semblent sous-tendus par l'obtention de lamelles présentant une morphologie et un module relativement normés.

Cinq lamelles sont transformées en outils de la famille Dufour dont trois sont appointées par une retouche directe distale. Le flanc droit de ces pièces correspond à un positif de face inférieure d'éclat (Fig. $6, n .^{\circ} 1$ à 3 ). Aucun nucléus n'est recensé, en revanche, la série comporte 5 éclats emportant l'essentiel des tables de débitage des nucléus (Fig. 6, n. $^{\circ} 9,11,12$ ). Ces pièces comportent toutes un large positif d'éclat sur leur flanc droit. Le «plein débitage» semble précédé par une première phase durant laquelle une première série lamellaire va structurer le volume du nucléus à l'aide d'enlèvements couchés obliquement par rapport à la future table de débitage (Fig. $6, n . .^{\circ} 4,5$ ). Dans un deuxième temps, une série lamellaire va être extraite à la jonction de la table et des enlèvements obliques. Ces supports ont une morphologie spécifique, reconnaissable par la présence en face supérieure d'une première série de négatifs fortement obliques vis-à-vis de l'axe de la lamelle, recoupée par un négatif présentant un axe identique au support. Ces produits relativement caractéristiques peuvent être dénommés lamelles à négatifs croisés ou simplement lamelles croisées (Fig. 6, n. 1 à 3 ).

La première série oblique a probablement un rôle de structuration générale du volume du nucléus, générant une convergence distale relativement accusée et cintrant l'un des flancs du nucléus, le flanc opposé étant naturellement délimité par la face inférieure de l'éclat, support à ce débitage (Fig. 7). Les lamelles croisées interviennent probablement avant l'extraction de lamelles à la jonction de la table de débitage et de la face inférieure de l'éclat/nucléus. Ces dernières lamelles possèdent une morphologie relativement normée: il s'agit de lamelles faiblement convexes présentant une unique arête centrale et dont un des flancs (apparemment plus couramment dextre) correspond à un positif d'éclat. Ces supports semblent les seuls employés dans le cycle de production des outillages retouchés et correspondent probablement à un plein débitage. A l'inverse, les lamelles obliques et croisées, moins normées, et surtout nettement plus convexes sont probablement considérées soit comme des déchets, soit comme des éléments de second choix, qui, quoi qu'il en soit, ne semblent pas correspondre aux normes des artisans en vue de la réalisation de leurs outillages retouchés.

Cette production de lamelles repose sur une notion de prédétermination dépendante à la fois d'une importante structuration dans la géométrie des nucléus et sur l'emploi de formules relativement standardisées. II n'y a donc pas de continuité possible entre ces débitages et la production de petites lamelles qui est caractérisée par une très faible standardisation des chaînes opératoires.

Les grandes lamelles rectilignes ressortent encore d'une autre dynamique. Avec cinquante et une pièces se rattachant à cette production, ce schéma est 


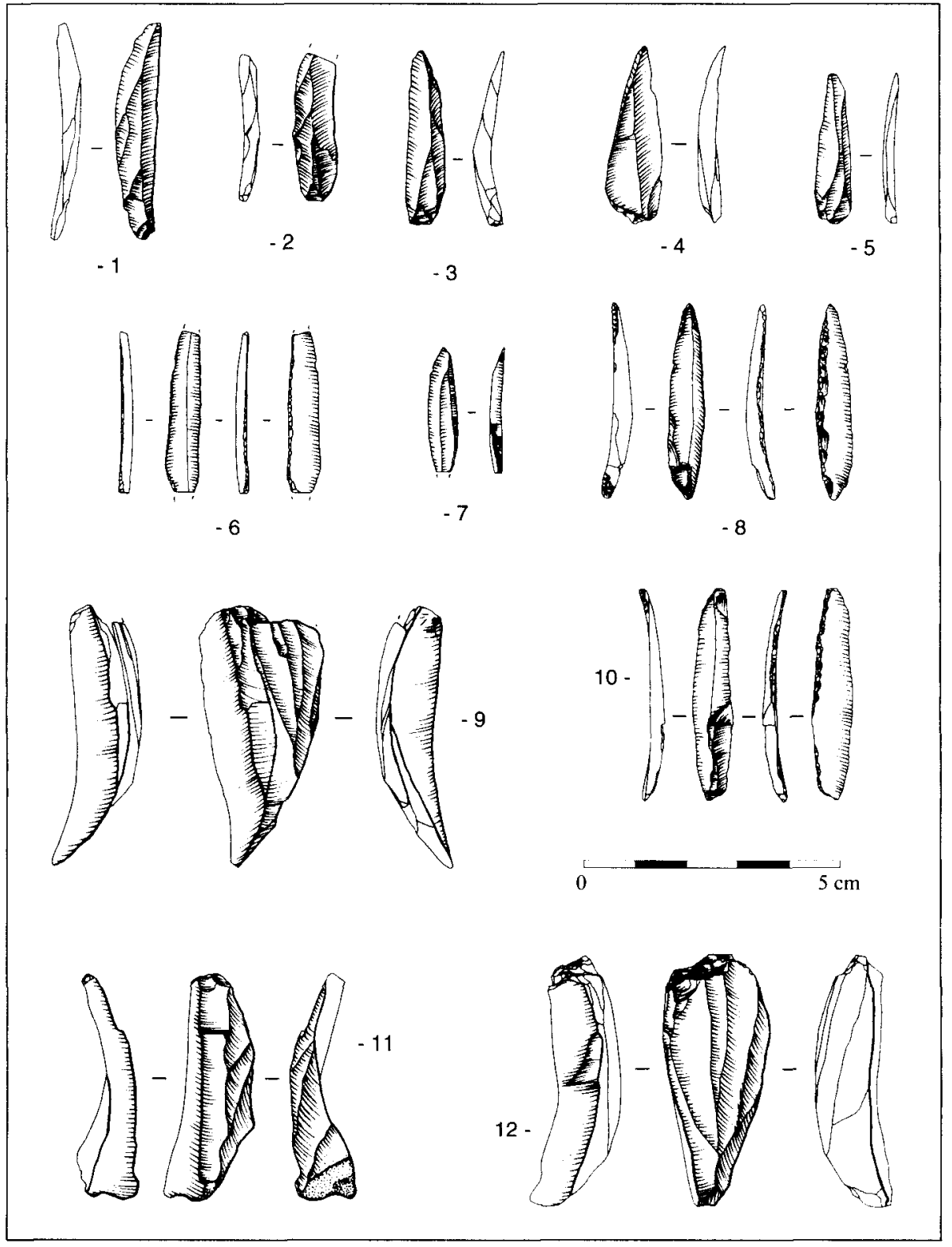

Figure 6. Eléments de la production de lamelles Dufour pointues.

proportionnellement le mieux représenté de la série. L'objectif du débitage semble être l'obtention de supports sveltes, réguliers dans leur morphologie et leur délinéation et particulièrement rectilignes. Le module recherché avoisine les $6 \mathrm{~cm}$ de longueur pour $1 \mathrm{~cm}$ de largeur. Cette production est dépendante d'un très fort 


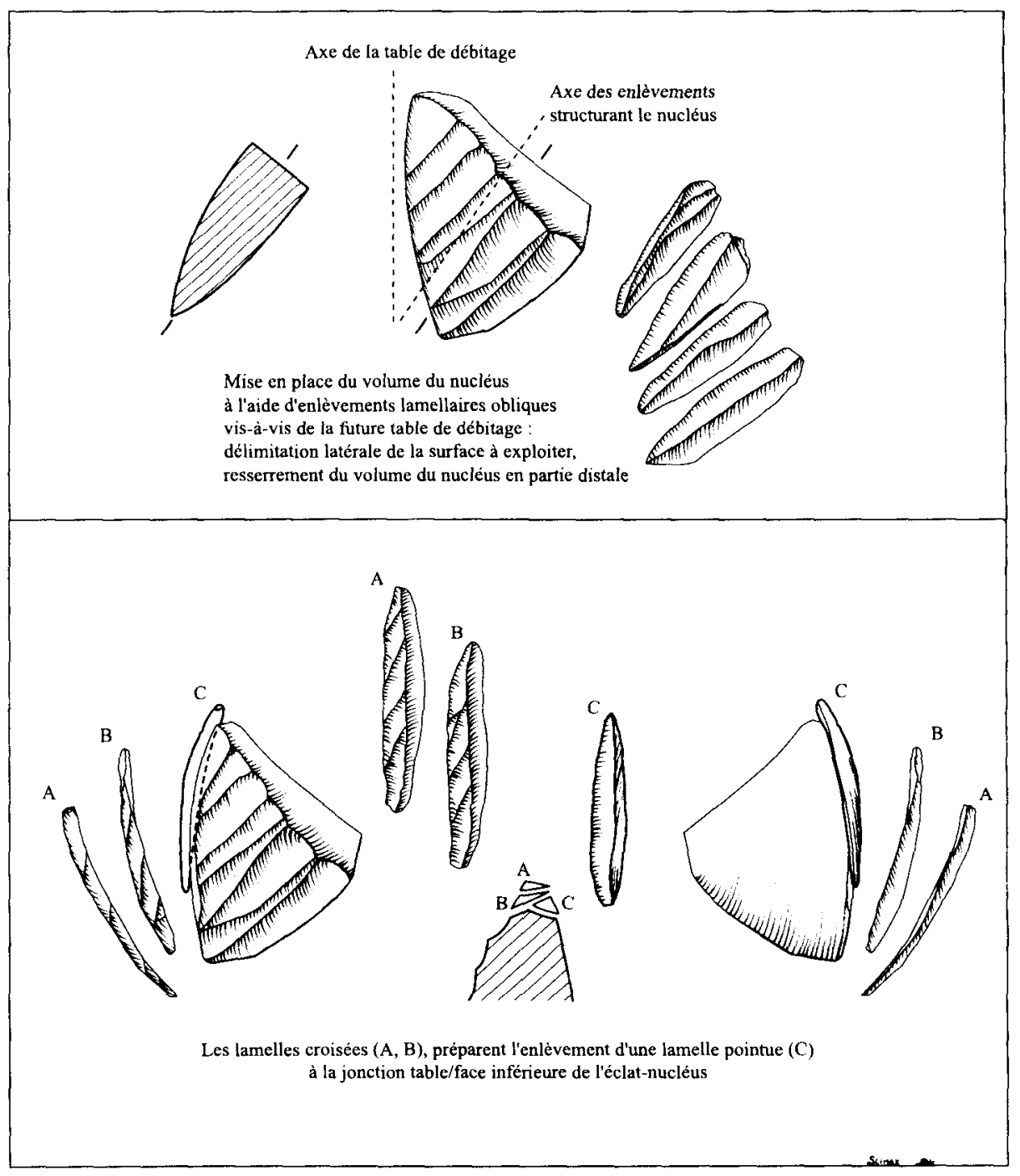

Figure 7. Schéma de production de lamelles Dufour pointues.

investissement dans la structuration géométrique du nucléus. La table de débitage est délimitée par deux larges flancs lisses qui correspondent soit à des positifs de faces inférieures, soit à de très larges négatifs d'éclats. Ces flancs cloisonnent latéralement la table de débitage et établissent un resserrement dans son tiers distal. Le dos du nucléus est aménagé par des larges enlèvements partant d'un des flancs du nucléus et outrepassant sur le flanc opposé. La table de débitage est délimitée dans sa partie distale par des enlèvements lamellaires opposés qui ont probablement un rôle de carénage de la surface exploitée (Fig. 10). 


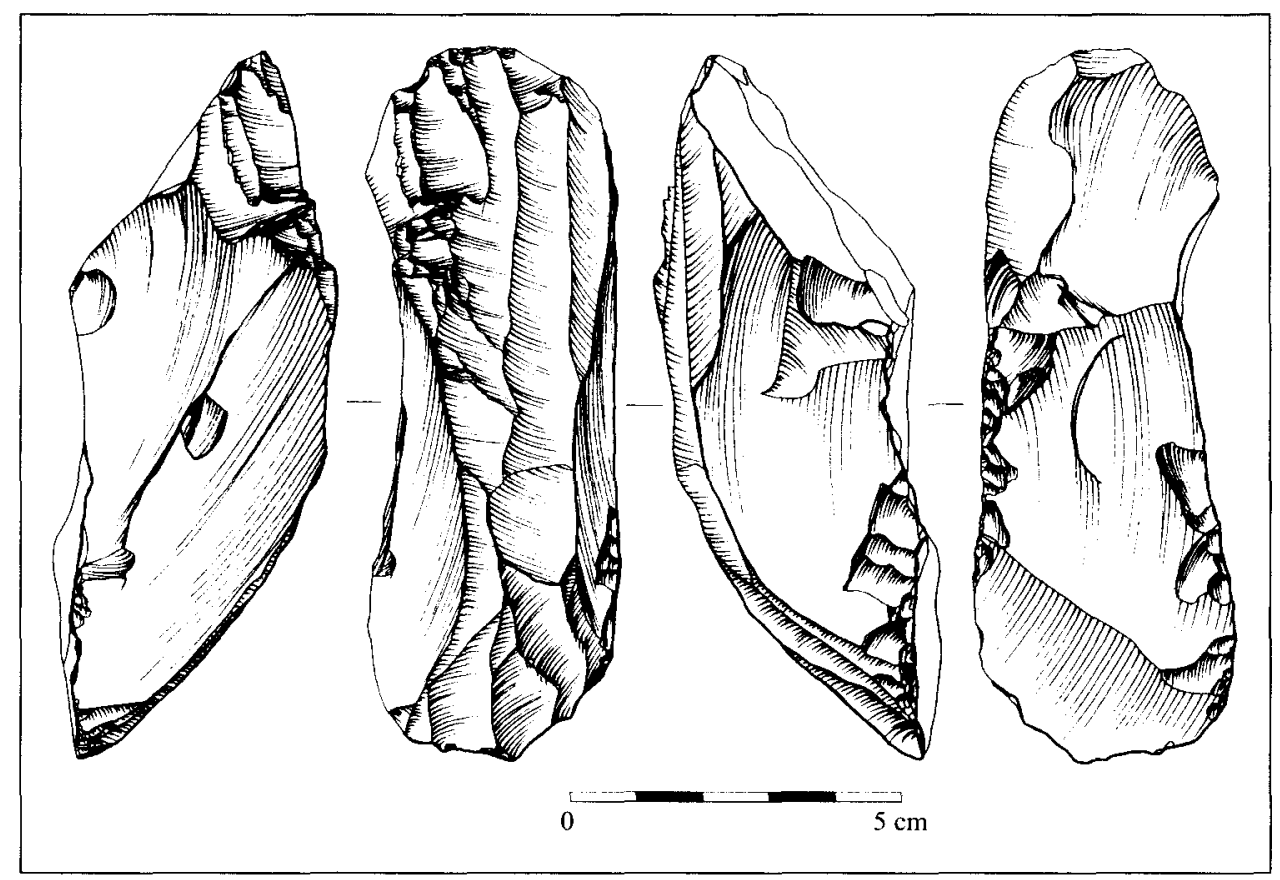

Figure 8. Nucléus à grandes lamelles Dufour.

Ces débitages sont donc dépendants de nucléus dont le volume est fortement structuré: délimitation latérale par des flancs lisses, resserrement de la surface exploitée dans son tiers distal, carénage assuré par des enlèvements opposés lamellaires, aménagement du dos du nucléus. La table de débitage est particulièrement plane et la géométrie globale du nucléus semble avoir pour finalité l'obtention de supports particulièrement rectilignes et d'épaisseur constante. La structure volumétrique des nucléus fournissant ces grandes lamelles est bien établie, et attestée par différents sous-produits qui indiquent l'exploitation de plusieurs blocs (un minimum de 5 ) suivant des modalités très proches, sinon identiques.

En revanche, les formules employées lors de l'exploitation de la table de débitage stricto sensu ne sont qu'imparfaitement restituables. La régularité des grandes lamelles paraît sous-tendue par une notion de standardisation de certains produits, notion antinomique avec un déroulement semi-tournant du débitage. La dynamique des différents enlèvements sur la surface exploitée met probablement en jeu des alternances de supports en vue de l'aplanissement de la table de débitage et de l'obtention d'un (ou de plusieurs) support rectiligne et d'épaisseur constante (Fig. 9, n. ${ }^{\circ}$ 2). Toutefois, les successions d'enlèvements établies par l'artisan ne sont pas directement restituables. 


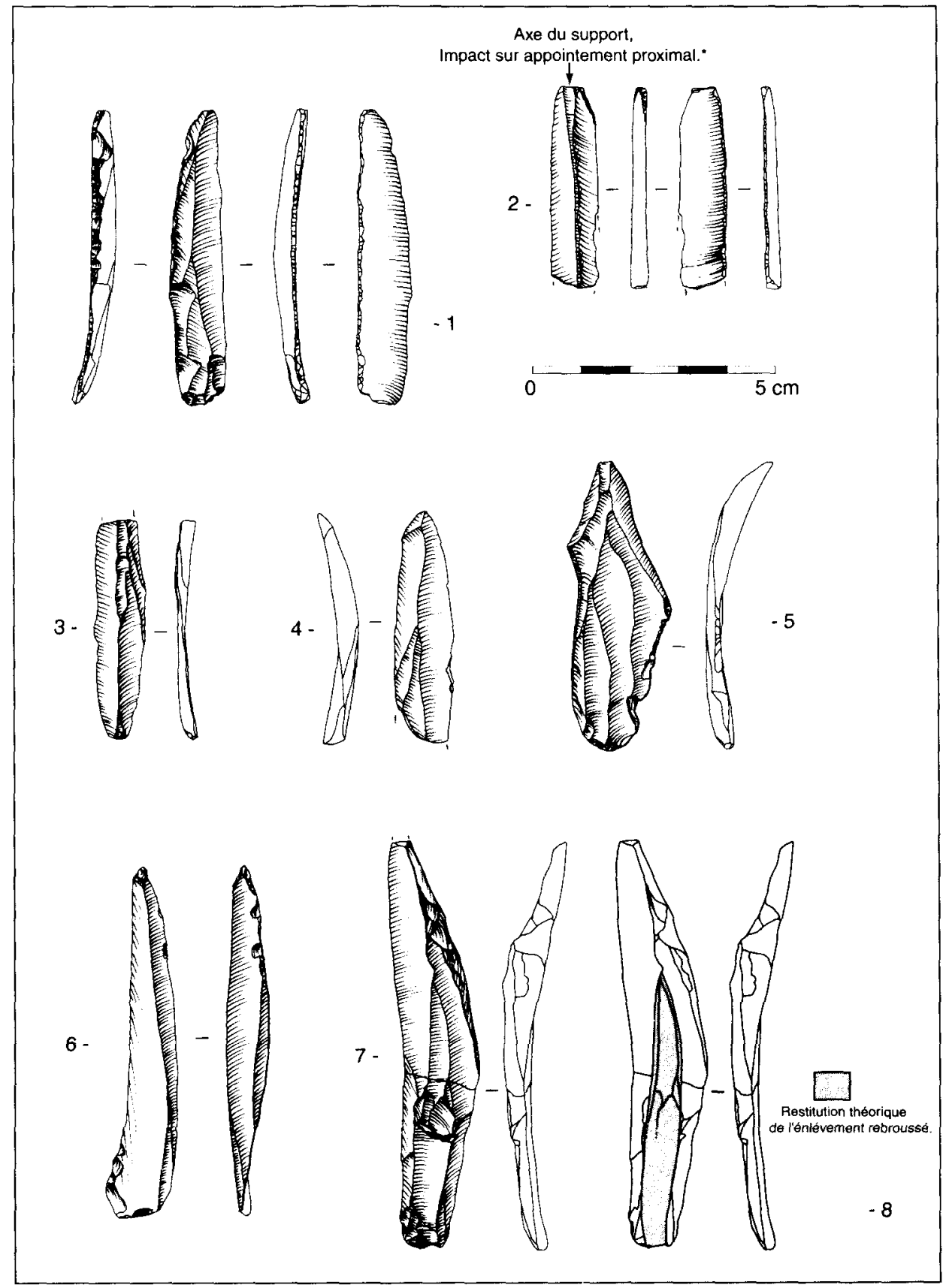

* Impact mis en évidence par F. Bon, confirmé par H. Plisson, comm. pers.

Figure 9. Eléments issus de la production de grandes lamelles. 
Quelques supports semblent indiquer l'emploi de formules proches de celles employées dans le cadre de la production des lamelles pointues. La présence de 2 lamelles croisées suggère une alternance entre des enlèvements obliques, prédéterminants, préalablement à l'extraction de lamelles croisées, rectilignes et axées sur la table de débitage (Fig. 9, n. ${ }^{1}$ et 4 ). Cette formule rappelle les algorithmes mis en jeu lors du débitage de lamelles pointues. En revanche, les objectifs sont nettement différenciés: au sein du débitage de lamelles pointues les lamelles croisées constituent probablement des déchets techniques, alors qu'elles correspondraient ici à un plein débitage ou, pour le moins, aux supports qui sont techniquement les plus investis.

Ce qui semble unifier ces deux familles de débitages lamellaires résulte donc de l'emploi de formules équivalentes, mais assujetties à des objectifs techniques différents.

II faut dès lors relever dans l'assemblage la coexistence de supports morphologiquement proches mais obtenus au sein de schémas nettement différenciés: ni le moment de leur obtention, ni leur finalité ne sont directement comparables. Cependant, les lamelles croisées mettent en jeu des formules communes qui pourraient stigmatiser un «tour de main» propre à un artisan ou à un groupe. Par ailleurs, la structure géométrique particulièrement complexe des nucléus à grandes lamelles individualise définitivement cette production des débitages de lamelles pointues. Si certaines formules élaborées dans la dynamique du débitage peuvent être communes, aucune continuité opératoire n'est possible. Les objectifs du débitage sont d'ailleurs trop fortement différenciés pour envisager des passages entre ces différents schémas. La très forte structuration des nucléus propre à la production de grandes lamelles est très probablement générée par des objectifs de production à la fois rigides et fortement prédéterminés, c'est-à-dire dont chacun des paramètres morphologiques et métriques sont connus et recherchés.

La rigidité du processus opératoire et la standardisation technique sous-jacente permettent de replacer de manière précise chaque produit sur un nucléus dont ils ne proviennent pas (Fig. 10). II s'agit là d'un remontage presque physique tant les produits reflètent les caractères structuraux de ce nucléus à grandes lamelles Dufour 5. II faut relever en dernier lieu que l'abandon du nucléus à grandes Dufour intervient précocement dans le déroulement de la chaîne opératoire, à la suite d'une série d'enlèvements rebroussés, ces négatifs correspondant probablement à la mise en place «d'enlèvements croisés". Ces rebroussés ne sont cependant pas rédhibitoires dans l'éventualité d'une poursuite du débitage, en revanche, la continuation du débitage aurait nécessité une restructuration locale de la géométrie du nucléus et surtout une modification dans sa dynamique d'exploitation. La progression du débitage nécessitait alors l'emploi de formules déviant légèrement vis-à-vis du schéma conceptuel de l'artisan. L'abandon en l'état d'un nucléus fortement investi

${ }^{5}$ Cette démarche de visualisation graphique d'éléments issus d'un même schéma de débitage mais pouvant provenir de différents blocs constitue une "mise en situation "(Slimak 2004) qui représente l'expression graphique d'un remontage mental (Pelegrin 1986). 


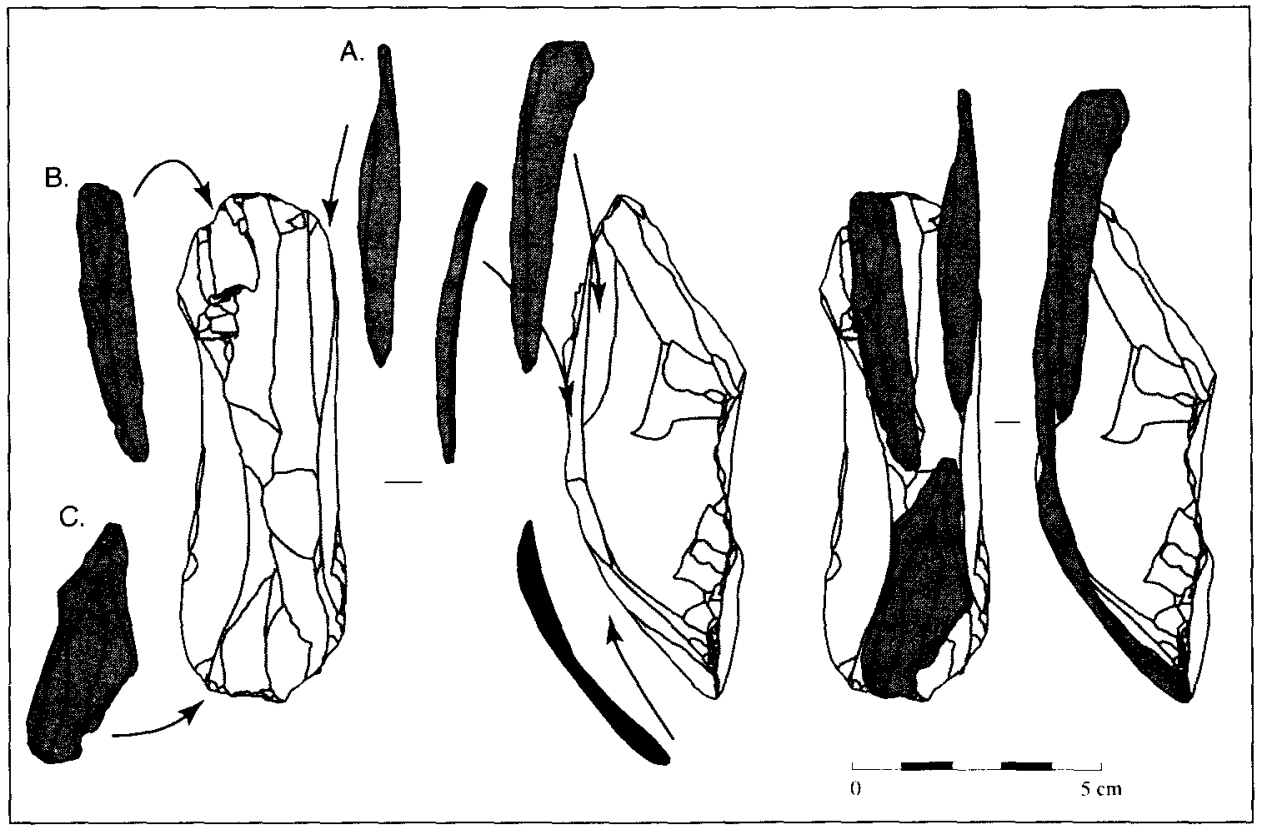

Figure 10. Proposition de restitution des éléments de la figure 9 résultant de la production de grandes lamelles:

Mise en situation des éléments appartenant à un même schéma de production, maix issus de quatre blocs différents. (Tous les éléments sont réduits à $75 \%$ ).

Le suport débordant (A.) intervient dans une phase très antérieure (mise en forme ? exploitation ?) du nucléus. La grande Dufour (B.), correspond à une représentation en miroir de la pièce en Fig. 9, n. ${ }^{\circ}$. Cette mise en situation permet d'ilustrer la forte identitéentre les négatifs croisés du support et les enlèvements latéraux rebroussés visibles sur le nucléus.

L'élément (C.) est une reprise de carène par enlèvement opposé. Cette pièce présente dans sa partie distale des négatifs provenant du plan de frappe principal et emporte latéralement un positif ou un négatif de très large enlèvement correspondant au flanc du nucléus.

Il faut relever l'adéquation à la fois morphologique, métrique de ces diférents produits vis-à-vis du nucléus. Ainsi, la rupture de profil dans le tiers distal de la table de débitage du nucléus, se retrouve à quelques près sur l'enlèvement (C.). Cette homologie induit une très forte standardisation de ces débitages.

dans sa conception et dans un matériau local mais d'excellente qualité induit une forte standardisation des formules employées dans l'élaboration de ces débitages.

\subsection{Les productions laminaires}

Les débitages laminaires ressortent d'une toute autre dynamique. Ces débitages sont globalement peu représentés dans la série, avec seulement 26 pièces (pour une série comprenant 162 éléments). Deux entités semblent coexister avec d'un côté de petites lames faiblement élancées et peu normées, 
constituant l'essentiel du corpus laminaire, et de l'autre un élément laminaire de grand module, témoignant apparemment de la production de supports laminaires massifs et probablement fortement normés. Cette production n'est cependant représentée que par deux éléments se raccordant et probablement emmenés dans la grotte sous forme de produits finis (Fig. 11, n. ${ }^{\circ}$ ).

Les petites lames semblent résulter de modalités de débitage sous-tendues par une recherche de supports globalement peu normés relevants de modalités de débitage simples. Les nucléus sont probablement peu investis, le débitage s'amorce depuis une crête à deux versants, voire à partir de l'extraction du dièdre naturel d'un bloc. Le débitage s'élabore alors suivant un mode semi-tournant lato sensu générant une forte proportion de supports laminaires présentant un flanc cortical (11 lames à flanc cortical pour 24 supports laminaires) (Fig. 11, n. ${ }^{\circ}$ 4). L'extraction de lamelles intercalées est illustrée par un remontage (Fig. 11, n. ${ }^{\circ}$ 1à 3) et une restitution (Fig. 9, n. ${ }^{\circ}$ 7) mais, en l'état, il n'est pas possible de savoir à quel(s) moment(s) intervient cette production. Une lame permet de supposer l'emploi (épisodique?) d'un plan de frappe opposé (Fig. 11, n. ${ }^{\circ} 5$ ).

Le débitage laminaire occupe donc une place dans l'assemblage à la fois marginale et techniquement peu investie. Ces débitages sont donc globalement porteurs des informations les moins diagnostiques de cette série: un débitage globalement unipolaire très faiblement structuré et présentant probablement une certaine variabilité technique. Cette très faible structuration du débitage individualise définitivement ces productions des deux principaux schémas lamellaires (lamelles pointues et grandes lamelles) qui sont très fortement structurés en vue de l'obtention de supports particulièrement normés. Il est donc impossible d'envisager la moindre continuité opératoire entre débitages laminaires et lamellaires. Le débitage laminaire qui n'occupe qu'une place marginale de l'assemblage paraît résulter d'une production presque expéditive de supports peu normés qui ne sont d'ailleurs pas intégrés à un cycle de transformation en outillage: l'outillage sur lame comprend au total deux grattoirs, l'un étant sur petite lame, l'autre sur la grande lame. La retouche «aurignacienne» n'est pas représentée.

\subsection{Conclusion}

Cette présentation, certes qualitative, met en évidence des procédés de débitage qui ne présentent que de faibles recoupements entre eux et sont générés par des logiques productives fortement dissociées. S'il peut parfois exister une incertitude quant au rattachement de telle ou telle pièce à un schéma déterminé, celle-ci est exclusivement le fruit de convergences morphologiques qui n'ont aucun corollaire en ce qui concerne les schémas de débitage et les objectifs de la production.

Si chaque schéma productif n'est représenté que par peu d'éléments, la diversité faciologique des matériaux exploités permet d'asseoir chaque mode 


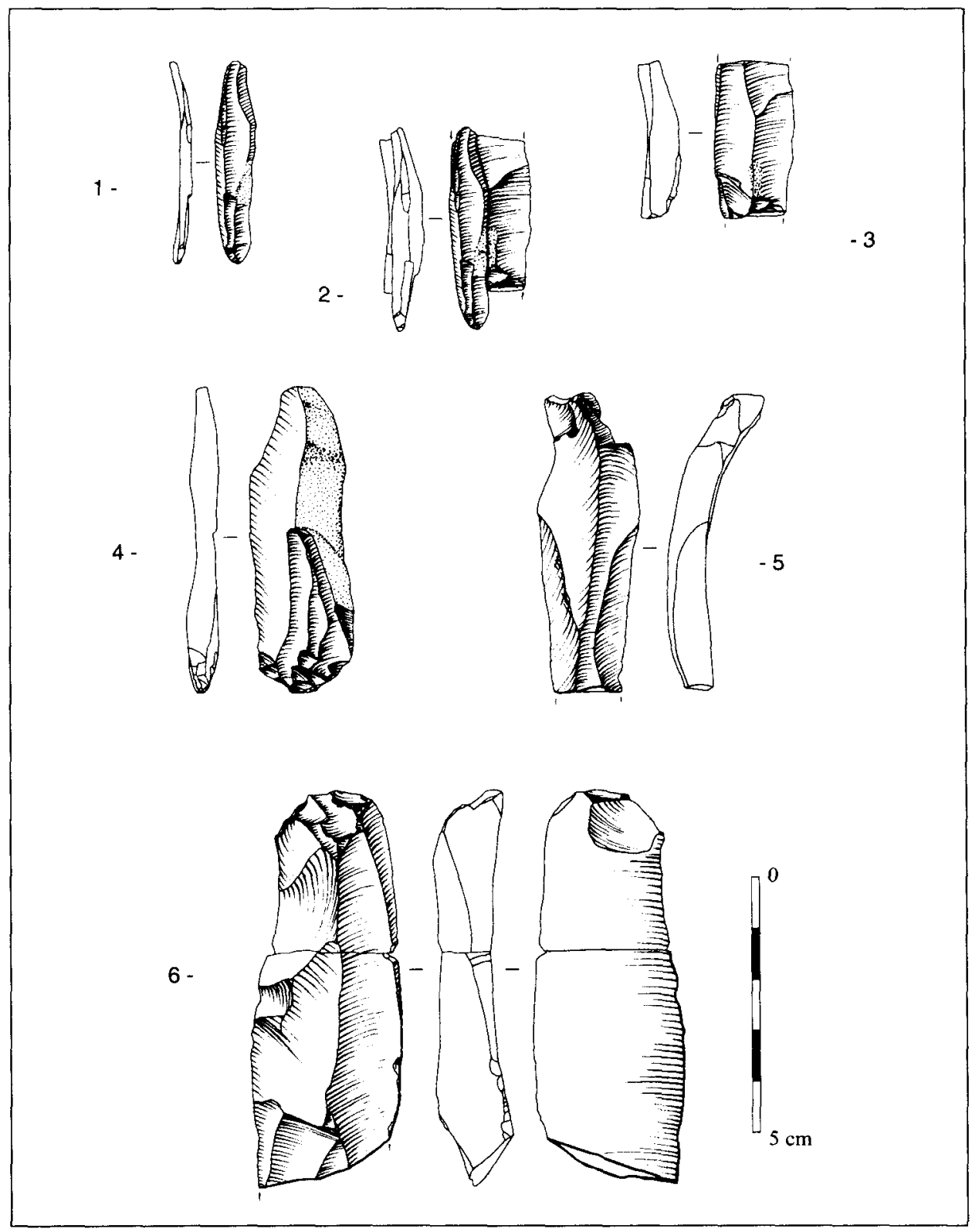

Figure 11. Remontage d'une lamelle intercalé $(1,2,3)$ et supports laminaires $(4,5)$ et grande lame raccordé (6).

opératoire sur un corpus de plusieurs blocs débités suivant des modalités très proches. Si la série est numériquement modeste, sa très forte homogénéité est en revanche un atout concernant la compréhension systémique de cet assemblage archéologique. 


\section{COMPARAISONS RÉGIONALES}

La série lithique de la grotte Mandrin présente donc des caractéristiques techniques très spécifiques, tant par les modalités du débitage que par les objectifs sous-jacents. L'obtention de lamelles rectilignes de grand module transformées en lamelles Dufour organise les activités de débitages décrites précédemment. Ces aspects sont en net décalage avec les productions de l'Aurignacien ancien (Bon 1993, Klaric 1999, Bon 2000, Bordes 2003). Toutefois, ces productions spécialisées ne demeurent pas isolées dans le contexte régional.

Dans tout le bassin rhodanien, seuls trois gisements présentent une occupation du Protoaurignacien: l'Esquicho Grapaou et la Laouza dans la vallée du Gardon et l'abri des Pêcheurs dans la vallée du Chassezac, principal affluent de l'Ardèche. Dans l'Ardèche même, qui constitue une des zone de plus forte densité en gisements pléistocènes, aucune occupation protoaurignacienne n'est clairement attestée. Seule la grotte du Figuier (niveau 2) et la grotte d'Oullins (niveau 2) présentent une petite installation aurignacoïde mais probablement tardive (Combier 1967). Ces deux gisements ardéchois ne s'inscrivent donc pas directement dans la problématique des premières occupations du Paléolithique supérieur. Les autres points de comparaison se trouvent hors du bassin rhodanien. II s'agit de la grotte Rainaude dans le Var (Onoratini, 1986) et des gisements du "groupe de Grimaldi» (Cheynier 1965) comprenant l'abri Mochi (Kuhn et al. 1998) et la grotte de l'Observatoire (Boule et al. 1927).

Les sites du Gardon constituent des points de comparaisons privilégiés, par leur proximité géographique et la nature des assemblages, décrits par S. Sicard (1994, 1995). La production de grandes lamelles Dufour organise largement les activités de débitage, comme nous avons pu l'observer pour la grotte Mandrin. Cependant, même si d'un point de vue morphométrique les objectifs de débitage sont proches, les modalités de cette production sont fort différentes. Par contre certains procédés de débitage se retrouvent à Mandrin comme à la Laouza, notamment la production de petites lames sur blocs peu préparés et l'extraction de supports lamellaires au cours de séquences laminaires (Sicard, 1995). Néanmoins ces débitages constituent à Mandrin les procédés les moins investis techniquement et peuvent être considérés comme peu diagnostics. En revanche l'outillage laisse supposer l'existence de liens entre les assemblages gardois et la Grotte Mandrin. La recherche de deux modules de lamelles Dufour, sous-type Dufour, a été mise en évidence dans les deux gisements languedociens: un module de moyennes Dufour souvent un peu arquées et un module de grandes lamelles Dufour rectilignes, supérieures à $5 \mathrm{~cm}$. Dans ces industries languedociennes, les débitages lamellaires occupent une place très importante. L'outillage de la Laouza se compose de $35 \%$ de lamelles retouchées, et atteint $60 \%$ à l'Esquicho Grapaou (Sicard, 1995, 1994). II ne peut s'agir ici d'une simple convergence entre gisements. La place des productions lamellaires et la nature de la transformation des supports peuvent être interprétées comme des caractères forts et structurants les activités de production et de consommation de ces trois gisements. 
Cette surreprésentation de l'outillage lamellaire n'est pas anecdotique et se retrouve sur l'ensemble des gisements attribués à l'Aurignacien initial méditerranéen. Dans les grottes de Grimaldi, le niveau G de l'abri Mochi comprend, selon les auteurs, 35\% de lamelles retouchées (Onoratini et al. 1999), 24,9\% pour la série 1938-49 et 30,9\% pour la série 1959 (Kuhn et al. 1998). Dans la petite série de l'Observatoire, les lamelles retouchées représentent la moitié de l'outillage des niveaux $\mathrm{F}$ et $\mathrm{G}$ (Onoratini et al. 1999).

La nature de la production lamellaire ne constitue pas le seul dénominateur commun à ces ensembles méditerranéens. L'élément le plus notable étant l'absence de fonds commun aurignacien: les séries comprennent systématiquement peu de grattoirs par ailleurs rarement carénés, et les lames massives de facture aurignacienne ne sont pas attestées. La retouche aurignacienne n'est pas représentée dans ces séries (Bazile 1976 b, Bazile et al. 1999). Ces premières occupations du Paléolithique supérieur dégagent donc une certaine impression d'homogénéité mais qui ne doit pas masquer les particularités de chacun de ces assemblages.

L'ensemble des gisements protoaurignaciens du sud-est de la France sont exclusivement reconnus en grotte et abri, fréquemment au sein d'importantes séquences qui permettent de documenter avec une certaine précision leur position chrono-stratigraphique.

Superposé à ce Protoaurignacien, se rencontre un Aurignacien ancien aux Pêcheurs (Lhomme, 1976), à l'Esquicho Grapaou, niveaux B.R. 1 et C.C. 1 (Bazile 1977), à Mochi, niveau F (Kuhn et al. 1998) et à l'Observatoire niveau E (Onoratini et al. 1999). Cet Aurignacien ancien se caractérise par l'abondance des grattoirs, notamment de carénés et des lames aurignaciennes. En outre, il voit l'apparition de la sagaie à base fendue, principal fossile directeur de l'Aurignacien I, aux Pêcheurs (Fig. 12), à l'abri Mochi et à la grotte de l'Observatoire.

Toutefois, une différenciation entre Protoaurignacien et Aurignacien ancien ne permet pas d'envisager de manière dynamique les premières phases de l'Aurignacien dans cette aire géographique. En effet, certains assemblages viennent nuancer cette dichotomie.

Dès 1966, G. Laplace distingue plusieurs phases durant le Protoaurignacien, selon la représentation relative des grattoirs et des lamelles Dufour. Cette distinction fut reprise par G. Onoratini (Onoratini 1985, 1986, Onoratini et al. 1999) à partir de la couche 10 de la grotte Rainaude. Cette série présente des caractères typologiques considérés comme intermédiaires entre le Protoaurignacien et l'Aurignacien ancien: les lamelles Dufour ne représentent que 14\% de l'outillage et sont associées à une importante série de grattoirs $(20 \%)$, dominée par des éléments carénés et à museau (Onoratini 1986). La représentation des grattoirs démarque cet ensemble de Mandrin, de la Laouza et des sites de Grimaldi. Seul l'Esquicho Grapaou, qui comprend une fraction laminaire légèrement plus 


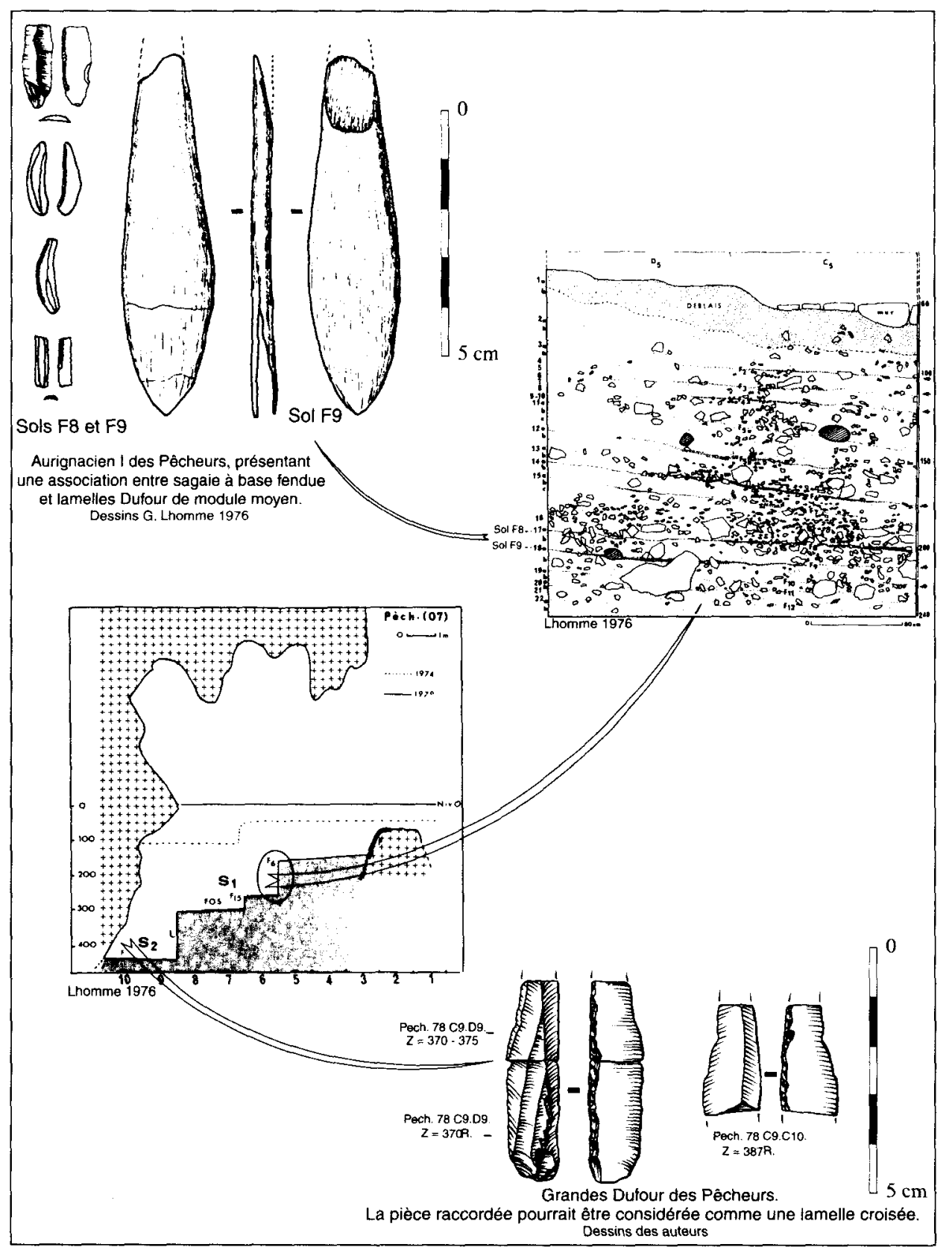

Figure 12. Abri des pêcheurs, localisation stratigraphique de la sagaie à base fendue (Sol F9) et des grandes Dufour. Les grandes Dufour sont séparées du niveau à sagaie par un remplissage de plus de $150 \mathrm{~cm}$. 
importante, présente un pourcentage de grattoirs de 9,9\%. Enfin, la retouche écailleuse, qui constitue un des caractères identitaires de l'Aurignacien n'est représentée que dans le niveau 10 de la grotte Rainaude. Ces éléments amènent l'auteur à situer cette occupation dans une phase plus récente (évoluée?) que la couche $G$ de Mochi et que la Laouza. F. Bazile interprète de manière similaire la timide apparition de la retouche latérale sur les supports laminaires de l'Esquicho Grapaou en supposant que cette retouche puisse "traduire un caractère évolutif» des sites protoaurignaciens (Bazile et al., 1999).

Dans cet ordre d'idées, il semblerait que des caractères de l'Aurignacien ancien apparaissent dans certains assemblages du Protoaurignacien et que des éléments protoaurignaciens perdurent dans les séries de l'Aurignacien ancien méditerranéen. Dans l'aire géographique concernée, plusieurs ensembles rattachables à un Aurignacien ancien présentent des lamelles de module plus important que celui observé dans l'Aurignacien ancien du sud-ouest de la France. Ces lamelles appartiennent au sous-type Dufour et correspondent globalement au module moyen mis en évidence dans les assemblages méditerranéens. A ces éléments sont associées des sagaies à base fendue aux Pêcheurs (sols $F 8$ et $F 9$ ) (Fig. 12), à Mochi (niveau F) et à l'Observatoire (niveau E).

II en ressort que si dans l'aire géographique concernée l'antériorité du Protoaurignacien méditerranéen sur l'Aurignacien I paraît établie, il faut émettre l'hypothèse de liens phylétiques entre ces deux ensembles. A un Protoaurignacien, représentant la première vraie expression du Paléolithique supérieur succèderait un Aurignacien ancien à connotation méditerranéenne.

\section{CONCLUSION}

La reconnaissance à la grotte Mandrin de schémas de production fortement normés apporte de nouveaux éléments quant à la compréhension des premiers groupes du Paléolithique supérieur. Si, compte tenu de la faiblesse de l'effectif et d'un approvisionnement en matière première très ciblé, peu de données économiques ressortent de cette analyse, l'identification et l'individualisation de plusieurs modes de débitages laminaires et lamellaires constitue un référentiel important dans une optique de reconsidération de l'Aurignacien initial.

Les gisements méditerranéens partagent plusieurs caractères: prédominance des débitages lamellaires en vue de l'obtention de lamelles Dufour de moyen et grand module, faible représentation des outillages laminaires et enfin, rareté du fonds commun Aurignacien. La récurrence de ces caractères pourrait être perçue comme une véritable "structure protoaurignacienne". Il ne s'agit donc pas à proprement parler d'un Aurignacien "archaïque" (Sicard, 1994), mais bien d'un groupe pleinement constitué (Bazile et al. 1999), méritant certainement, comme le propose cet auteur, d'être individualisé sous une appellation qui lui soit spécifique. 
Comme le suggère $F$. Bazile, le terme de Mochien proposé par $A$. Cheynier en 1965 pourrait être un candidat valable dans son acceptation réduite de Mochien méditerranéen, notion qui recouperait la notion d'Aurignacien initial méditerranéen de F. Bazile (1999).

L'éventualité de liens phylétiques à caractère méditerranéen entre Protoaurignacien et Aurignacien I souligne la nécessité de développer, dans un premier temps, des problématiques régionales avant d'élargir le champ des comparaisons à un espace géographique plus large.

\section{BIBLIOGRAPHIE}

BAZILE, F. (1974): Nouvelles données sur le Paléolithique supérieur ancien en Languedoc oriental, $X X^{\circ}$ Congrès Préhistorique de France: 24 à 28.

- (1976 a): Datations absolues sur les niveaux paléolithiques supérieurs anciens de la grotte de l'Esquicho Grapaou (Sainte-Anastasie, Gard), Bulletin de la Société Préhistorique Française, 73: 205 à 207.

- (1976 b): Recherches sur le Paléolithique supérieur ancien «présolutréen" en Languedoc oriental, Bulletin de la Société Préhistorique Française, 73: 226 à 227.

- (1977): Recherches sur le passage du Würm ancien au Würm récent et sur le début du Würm récent en Languedoc oriental, Mémoire de Doctorat, Université Paul Valéry de Montpellier.

BAZILE, F., SiCARD, S. (1999): Le premier Aurignacien du Languedoc oriental dans son contexte méditerranéen, in Les faciès leptolithiques du Nord-ouest méditerranéen: milieux naturels et culturels, XXIV ${ }^{\circ}$ Congrès Préhistorique de France, Actes du colloque international de Carcassonne du 26 au 30 septembre 1994, sous la direction de D. Sacchi, Société Préhistorique française.

Bon, F. (1993): L'industrie lithique aurignacienne de la couche 2 a de la grotte des Hyènes à Brassempouy (Landes). Mémoire de Maîtrise. Université de Paris 1.

- (2000): La question de l'unité technique et économique de l'Aurignacien: réflexions sur la variabilité des industries lithiques à partir de l'étude comparée de trois sites des Pyrénées françaises (la Tuto de Camalhot, Régismont-le-Haut et Brassempouy), Mémoire de Doctorat, Université de Paris 1.

BoRDES, J.-G. (2003): Les interstratifications Châtelperronien/Aurignacien du Roc-de-Combe et du Piage (Lot, France). Analyse taphonimique des industries lithiques; conséquences archéologiques. Mémoire de Doctorat. Université de Bordeaux.

Boule, M., VIlleneuve, L. (1927): La grotte de l'Observatoire à Monaco. Archives de l'Institut de Paléontologie Humaine, Mémoire 1.

Cailleux, A.: Code des couleurs des sols, Notice (15 pag.) + code.

CHEYNIER, A. (1955): Lettre ouverte à M. Angelroth suite à sa publication sur le Périgordien et l'Aurignacien en Belgique, Bulletin de la Société Préhistorique Française: 52: 515 à 517.

- (1965): Comment vivait l'Homme des cavernes à l'Âge du Renne?. Editions du Scorpion.

COMBIER, J. (1960): La structure du Paléolithique supérieur dans la région du Rhône moyen, Compte Rendu de l'Académie des Sciences, 250: 1889-1891.

- (1967): Le Paléolithique de l'Ardèche dans son cadre paléoclimatique. Bordeaux, Delams, Mémoire de. l'Institut de Préhistoire. Université de Bordeaux.

- (1990): De la fin du Moustérien au Paléolithique supérieur. Les données de la région rhodanienne, in: Farizy C. dir., Paléolithique moyen récent et Paléolithique supérieur ancien. Ruptures et transitions: examen critique de documents archéologiques, Nemours 1990, Mémoire du Musée de Préhistoire d'lle-de-France, 3, APRAIF: 267-278.

Giraud, Y., Brugal, J.-P., JEANNET, M. (1998): Un nouveau gisement moustérien en moyenne vallée du Rhône: la grotte Mandrin à Malataverne (Drôme), Bulletin de la Société Préhistorique Française, 95 (1): $7-15$.

Giraud, Y. (1999): Grotte Mandrin, Malataverne (Drôme), Rapport de fouilles programmées pluriannuelles, 1997-1999.

KLARIC, L. (1999): Le site de Garret à Serreslous-et-Arribans (Landes): un gisement aurignacien de plein air, Archéologie des Pyrénées Occidentales et des Landes, 18: 101 à 112. 
KUHN, L. S., Stiner, C. M. (1998): The Earliest Aurignacian of Riparo Mochi (Liguria, Italy), Current Anthropology, 39, Supplement, June: 175 à 189.

LAPLACE, G. (1966): Recherche sur l'origine et l'évolution des complexes leptolithiques. Ecole Française de Rome, Mélanges d'Archéologie et d'Histoire, suppl. 4.

LHomme, G. (1976): Un nouveau gisement paléolithique en Ardèche: l'abri des Pêcheurs à Casteljau, premiers résultats, Etudes Préhistoriques, 13: 1 à 8.

OnORATINI, G. (1985): Les industries du Paléolithique supérieur ancien en Provence orientale et leurs relations avec celles de l'Italie, Etudes Quaternaires Languedociennes, cahier 4: 33 à 43.

- (1986): Découverte en Provence orientale (grotte Rainaude) d'une industrie souche de l'Aurignacien. Cette civilisation est-elle monolithique?, Bulletin de la Société Préhistorique Française, 83: 240-256.

OnORATINI, G., Simon, P., Simone, S. (1999): Mise en évidence du Protoaurignacien à la grotte de l'Observatoire (Principauté de Monaco), Bulletin du Musée d'Anthropologie préhistorique de Monaco, 40: $43-56$.

Pelegrin, J. (1986): Technologie lithique: une méthode appliquée à l'étude de deux séries du Périgordien ancien (Roc-de-Combe, couche 8 - la Côte, niveau III). Mémoire de Doctorat, Université de Paris X.

SICARD, S. (1994): L'Aurignacien archaïque de l'Esquicho-Grapaou: analyse typo-technologique du débitage. Mémoire de Maîtrise, Université de Paris I.

SICARD, S. (1995): La Laouza (Gard): approche techno-fonctionnelle d'une chaine opératoire aurignacienne. Mémoire de DEA, Université de Paris I.

SLIMAK, L. (2004): Les demières expressions du Moustérien entre Loire et Rhône. Mémoire de doctorat de l'Université de Provence.

YVORRA, P. (2003): The management of space in a Paleolithic rock shelter: defining activity areas by spatial analysis, Antiquity, 77 (296): 336 à 344.

YvorRA, P., Slimak, L. (2001): Grotte Mandrin à Malataverne (Drôme). Premiers éléments pour une analyse spatiale des vestiges en contexte moustérien, Bulletin de la Société Préhistorique Française, 98, (2): 189-205. 
